\title{
Vortex ring generation due to the coalescence of a water drop at a free surface
}

\author{
B. S. Dooley, A. E. Warncke, M. Gharib, G. Tryggvason
}

Abstract Results are presented of an experimental investigation of vortex ring formation by a fluid drop contacting a free surface with negligible velocity. The pool fluid is mixed with fluorescein dye, and a laser sheet is used to illuminate a plane of the flow. A series of representative images is recorded by a CCD camera and speculation is made regarding specific sources of vorticity flux through the free surface. Two scaling analyses previously presented by other investigators are demonstrated to be equivalent under the assumptions of this experiment, and they provide the motivation for a series of test runs in which the duration of the coalescence process, $\tau_{\star}$, is related to variations in drop diameter $L$ and fluid surface tension $\sigma$. Experimental results are in agreement with the analyses, showing $\tau_{\star} \sim \sigma^{-1 / 2}$ and $\tau_{\star} \sim L^{3 / 2}$.

\section{1}

\section{Introduction}

A fluid drop contacting a pool often generates a vortex ring which travels downward from the free surface, easily demonstrated with a milk drop and a glass of water. Studies of this phenomenon have been appearing in the scientific literature for well over a century. Rogers (1958) observes common characteristics among several processes of vortex ring generation, including a drop contacting a stagnant pool. Thomson and Newall (1885) examine in some detail the properties of vortex rings caused by falling drops of various liquids. For simplicity, much of the work done has been restricted to the case of the drop and pool having identical composition. Chapman and Critchlow (1967) characterize the rings formed

Received: 22 December 1995/Accepted: 15 October 1996

B. S. Dooley, A. E. Warncke, M. Gharib

Graduate Aeronautical Laboratories 205-45,

California Institute of Technology,

Pasadena, CA 91125, USA

\section{G. Tryggvason}

Department of Mechanical Engineering and Applied Mechanics, University of Michigan, Ann Arbor, MI 48109, USA

Correspondence to: M. Gharib

This work was partially supported by the Office of Naval Research, ONR-URI N00014-92-J-1610. Additionally, the second author received scholarship support from the National Science Foundation. Special thanks are due to Mr. S. Haase for help with the construction of the experimental apparatus and Dr. P. Atsavapranee for assistance with the photography. by falling drops in terms of the drop shape at the time of impact and propose a quantitative model of the vortex ring's generation process. Peck and Sigurdson (1994) analyze the structure of rings as they penetrate into the pool after a fall. Hsiao et al. (1988) experimentally determine a critical Weber number for the falling drop ( $W e \approx 8$ ) above which a jet, rather than a vortex ring, is formed in the pool. To further simplify matters, the degenerate case $(W e=0)$ in which contact between the drop and pool occurs with zero velocity may be examined. Shankar and Kumar (1995) observe the evolutionary behaviour of rings so generated, and characterize the zero velocity case as a function of two dimensionless parameters only. Cresswell and Morton (1995) examine falling drops with an eye toward explaining the mechanism of vorticity generation. They also seek to explain in detail the absence of vorticity in cases involving supercritical Weber numbers $(W e>8)$. In this work we visualize the formation of the ring rather than its evolution, and quantitatively examine a simplified scaling law based on the analyses of Hsiao et al., and Shankar and Kumar. This scaling law, when valid, allows the flow conditions to be expressed in a single dimensionless parameter. Validity, however, requires that surface tension forces dominate gravitational and viscous ones in the vortex ring formation process.

2

\section{Scaling}

If we consider only the case of the drop and pool fluids being the same, and assume that the drop must be spherical, then scaling laws may be readily found via dimensional analysis. Let the fluid density and surface tension be represented by $\rho$ and $\sigma$, respectively. Hsiao et al., in carrying out their scaling analysis, denote the surface energy time scale by

$\tau_{1}=\left(\rho L^{3} / \sigma\right)^{1 / 2}$

and the convective time scale by

$\tau_{2}=L / U$.

$L$ and $U$ are the drop diameter and velocity at time of impact, respectively. This scaling is still expected to be valid in the limit as $U$ goes to zero, leaving only $\tau_{1}$ finite. Our experimental setup enables a characteristic time of the vortex ring formation process to be measured, and we propose that this characteristic time $\tau_{\star}$ is directly proportional to the $\tau_{1}$ above. If this is the case, then it is valid to put the relationship between the relevant parameters in the form

$f\left(\frac{\tau_{\star}^{2} \sigma}{\rho L^{3}}\right)=0$ 
Such a relationship requires that $\tau_{*} \propto L^{3 / 2}$ and $\tau_{*} \propto \sigma^{-1 / 2}$. It must be noted that Hsiao et al. do not consider the effects of viscosity or gravity in the vortex ring formation, a simplification which requires relatively large surface tension effects. Our data indicate that this assumption is justified in some practical cases.

Shankar and Kumar, taking kinematic viscosity $v$ and gravitational acceleration $g$ into account, demonstrate that there can be two independent dimensionless parameters governing the flow. An analysis is then performed on the continuity and Navier-Stokes equations directly, using length and time scales $l$ and $\tau$, respectively. Gravity is assumed to act in the positive $x$ direction. Using carats to represent dimensionless quantities, the nondimensional form of the governing equations is

$\hat{\nabla} \cdot \hat{\mathbf{u}}=0$,

$\frac{D \hat{\mathbf{u}}}{D \hat{t}}=-\hat{\nabla} \hat{p}+\left(\frac{v \tau}{l^{2}}\right) \hat{\nabla}^{2} \hat{\mathbf{u}}+\left(\frac{g \tau^{2}}{l}\right) \hat{\mathbf{e}}_{x}$

$\hat{p}=\left(\frac{2 \sigma \tau^{2}}{\rho l^{3}}\right)\left(\hat{R}_{1}^{-1}+\hat{R}_{2}^{-1}\right)$

$\hat{R}_{i}$ are the (nondimensional) principal radii of curvature of the free surface. At first glance, it appears that three dimensional parameters have been obtained, but Shankar and Kumar point out that this is not the case. By setting $\tau=\sqrt{L / g}$ and $l=L$, one of the three becomes unity and we are left with only two independent parameters.

If, instead of following the lead of Shankar and Kumar, we consider the time scale $\tau$ to be a physical parameter of the problem and set $\tau_{\star}=\tau$, we then are left with three independent dimensionless parameters; $\alpha_{1}=\left(v \tau_{\star} / L^{2}\right), \alpha_{2}=\left(g \tau_{\star}^{2} / L\right)$, and $\alpha_{3}=\left(2 \sigma \tau_{\star}^{2} / \rho L^{3}\right)$. If we again neglect the effects of viscosity and gravity as small compared to those of surface tension, the functional relationship derived above from the work of Hsiao et al. is obtained.

\section{3}

\section{Apparatus and procedure}

The experiments were conducted in a Plexiglas test section $30 \mathrm{~cm}$ in height, having a $9 \mathrm{~cm}$ square cross section and an open top. Drops were formed at the end of a vertical glass pipette with an exterior diameter of $2.25 \mathrm{~mm}$ at the tip. The pipette was of standard, tapered, laboratory type, and was trimmed an appropriate distance from the original tip to yield the desired diameter. The wall thickness at the new tip was approximately $0.15 \mathrm{~mm}$. This pipette was rigidly held to a stand-mounted traverse to facilitate adjustment of the tip height above the surface of the pool. Drops were created through the use of a hand-operated syringe connected by a tube to the top end of the pipette. By careful adjustment of the height of the syringe above the free surface of the pool, drop size could be accurately controlled. Drops were very slowly "grown" at the end of the pipette using the syringe, and when the drop made contact with the free surface, a vortex ring was generated at the drop fluid was drawn into the pool. This technique is very similar to that used by Rogers (1858) in his experiments. Deionized water was used as the working fluid.
A mechanically shuttered laser sheet was created such that it illuminated a vertical plane in the test section passing through the drop's axis of symmetry. The laser sheet in the test section was approximately $0.5 \mathrm{~mm}$ thick. The solenoid-driven shutter was manufactured by NM Laser Products. Sheet alignment was done visually, with an estimated error of less than $0.5 \mathrm{~mm}$. Fluorescein dye was mixed into the pool fluid only for visualization purposes. The addition of dye to the fluid caused a measured reduction in surface tension of approximately $5 \%$.

A Texas Instruments Multi-cam CCD camera was initially positioned so as to look into the test section normal to the plane illuminated by the laser sheet. However, some experimentation led to the conclusion that the camera was best mounted to look down on the region of interest at an angle of approximately $8^{\circ}$. This positioning minimized optical distortion and line-of-sight blockage caused by the deformations of the free surface. The actual size of the camera's viewing area in the plane of the light sheet was approximately $5 \mathrm{~mm}$ square.

The camera was linked via a timing box with the shutter in the laser beam. This made it possible to obtain two $1 \mathrm{~ms}$ exposures as little as $3.5 \mathrm{~ms}$ apart (start-to-start). The camera had a frame rate of $1 / 30 \mathrm{~s}$; the two images were obtained very near the end of one frame exposure and just after the start of the next. Images were thus obtained in pairs; each pair was separated by approximately $65 \mathrm{~ms}$, far longer than the vortex ring formation process lasted.

The restriction to, at most, two images per individual drop was not a hindrance because of the high degree of repeatability of the vortex ring generation process. Multiple runs could be performed in succession, and a number of images obtained representing various stages of the vortex ring formation process. To acquire the images used in the analysis, many rings were formed while the camera captured random stages of their creation. After a sufficient number of runs, a full sequence was collected.

For such a collection of images to be useful, it was necessary to have a method of determining just when in the vortex ring formation process any given image was acquired. This was achieved by the use of an electrical circuit which was closed by the contact of the drop with the pool, and reopened when the liquid connection between the pool and pipette was broken. It proved necessary to add salt to the fluid to overcome the insulating qualities of deionized water.

The voltage across a resistor in the circuit was digitized and input to the Superscope software package (GW Instruments), along with the frame synchronization signal from the camera. For this application, Superscope was utilized only as a recording digital oscilliscope, triggering off the voltage rise at drop contact. After a run, a time plot of resistor voltage and the frame signal voltage was automatically displayed on the computer monitor. Drop contact and release times, as well as the times of the shutter openings, were manually read off the plot and recorded for each run. The rise time of the circuit was approximately $500 \mu \mathrm{s}$, but the moment of first contact between pool and drop was easily measured to within $300 \mu \mathrm{s}$, which represented the sampling period limit imposed by the software. Hence, all times so measured are accurate to $0.3 \mathrm{~ms}$.

The earlier statement that the process is very repeatable is based on the data taken as described above. Runs made consecutively, under the same experimental conditions, 
showed contact-to-release durations which varied by no more than approximately $3 \%$. This number represents a maximum; often, a series of runs showed no variation in duration, to the resolution of the time axis of the plot. This quantitative observation, plus the remarkable visual similarity of each run's plot to all others under similar conditions, justifies the assertion of repeatability.

The images from each run were stored in analog form on a write-once laser disc, and the frame number of each was recorded along with the information described above. A representative sequence of the ring generation process was collected by manually inspecting the image times and selecting an appropriate array. The required images could then be found on the laser disc and sorted chronologically.

A series of runs was performed in which the surface tension of the water was changed. This alteration was effected by the addition of progressively larger amounts of the surfactant sodium dodecylsulfate (SDS) to lower surface tension. In all cases, the fluid that was to make up the drop was drawn up into the pipette from the test section 5-10 min after the stirring in of the SDS, to minimize surface tension gradients between drop and pool fluid. This series of runs was done only after all other data had been taken. This allowed us to avoid concern with cleaning the plastic test cell to remove added contaminants.

Surface tension was measured with a Fisher Scientific ring tensiomat, and the uncertainty is thought to be less than $1.0 \mathrm{dyn} / \mathrm{cm}$. It was noted during the experiment that the surface tension of the fluid could decrease by up to $2 \mathrm{dyn} / \mathrm{cm}$ in times on the order of an hour, apparently due to contamination. It is not known whether this contamination came from the surrounding air or from the Plexiglas walls of the test section. To counter this, the surface of the test section fluid was vaccum-skimmed to remove contaminants regularly during the portions of the experiment in which surface tension was assumed to be held constant.

The drop diameter was measured manually off of a precontact image on a monitor. The tip of the pipette also appeared in the images, and its known diameter was used to properly scale the measurement. Some degree of prolateness was seen in the drops for almost every case; it became more pronounced as pipette tip height above the water surface increased. The "diameter" for the purposes of this experiment was taken to be the horizontal width of the drop. Uncertainty in the drop diameter measurements arise from estimated uncertainties in the pipette diameter and the measured image size on the monitor; the total error is thought to be less than $0.05 \mathrm{~mm}$, or about $2 \%$.

\section{Results}

\section{1}

\section{Visualization}

A sequence of 9 images was obtained as described above which is thought to accurately represent the vortex ring formation process. These images, along with one recorded just prior to contact, are shown in Figs. 1a-j. In this series, the drop diameter is approximately $3.3 \mathrm{~mm}$, and nothing has been added to the water except salt for conductivity and a small amount of dye in the pool fluid. As stated above, image exposure time was $1 \mathrm{~ms}$. Surface tension of the water was measured to be approximately $64 \mathrm{dyn} / \mathrm{cm}$ in this case. Kinematic viscosity and density were not measured, but meaningful variations from their standard values of $1.1 \times 10^{-6} \mathrm{~m}^{2} / \mathrm{s}$ and $998 \mathrm{~kg} / \mathrm{m}^{3}$, respectively, are not expected. The total time of the process, as measured by the closure time of the electrical circuit, is approximately $15.5 \mathrm{~ms}$. These images were recorded over the span of several hours; some variation in conditions was impossible to avoid. Circuit closure times varied by up to $6 \%$ from their median value among the images shown. This is not thought to significantly affect the qualitative analysis which follows. In all images, the source of the laser sheet is below and to the left, so there is some blockage of the light sheet in the region of interest on the left side of the image. We concern ourselves only with the right side of the these symmetric images.

The $1 \mathrm{~ms}$ exposure shown in Fig. $1 \mathrm{~b}$ actually captures the first instant of contact, as the circuit was not closed until about $800 \mu$ s into the light flash. Figure 1d shows the developing vortex roll-up, on the right side of the drop-pool interface. From this image, an order-of-magnitude estimate of fluid acceleration at the free surface may be obtained. The element of dyed fluid seen at the top of the roll-up (at the intersection of the free surface and the dyed/undyed fluid contact surface) is approximately $0.5 \mathrm{~mm}$ from initial pool surface position, from where it must have come. This image was taken roughly $3 \mathrm{~ms}$ after contact. If uniform acceleration from rest is assumed for discussion purposes, then the required surface-parallel acceleration on the fluid element is approximately $100 \mathrm{~m} / \mathrm{s}^{2}$.

In Figs. 1e-g, the vortex roll-up is observed to grow in size and number of visible coils as the ring formation process continues. Of note is the tendril of dyed fluid which continues to travel in an upward direction, above the primary vortex roll-up. This observation was also made by Cresswell and Morton in their experiments. Figure 2 shows a sketch of the tendril.

In Fig. $1 \mathrm{~h}$, the barest hint of a secondary roll-up can be seen at the upper extremity of this tendril of pool fluid. It is still visible in Fig.s $1 \mathrm{i}-\mathrm{j}$, although image distortion is becoming a problem by this point as the ring moves away from the surface. The distortion, present in all of the images, is a consequence of the $8^{\circ}$ down angle of the camera and the accompanying need to look through the highly deformed free surface.

\section{2}

\section{Sources of vorticity}

Based on the above images, it is possible to speculate as to the source terms of the vorticity which comes to be present in the fluid. As vorticity cannot be created away from the boundary of a body of homogeneous fluid, it follows that the roll-ups pointed out above must gain their vorticity via flux from the free surface. Rood (1994) presents a relation for vorticity flux, $v\left(\partial \omega_{\mathrm{z}} / \partial r\right)_{r=0}$, into a body of fluid through a curved free surface based on surface-fixed coordinates. This derivation supposes that the flow is two-dimensional and that the location of the free surface does not change with time. Neither is the case in this experiment, but we feel that the formulation is qualitatively relevant to this analysis. 


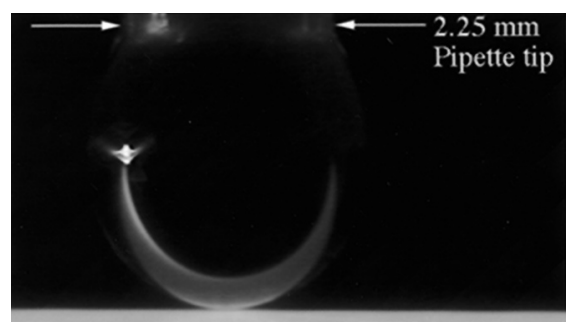

1a
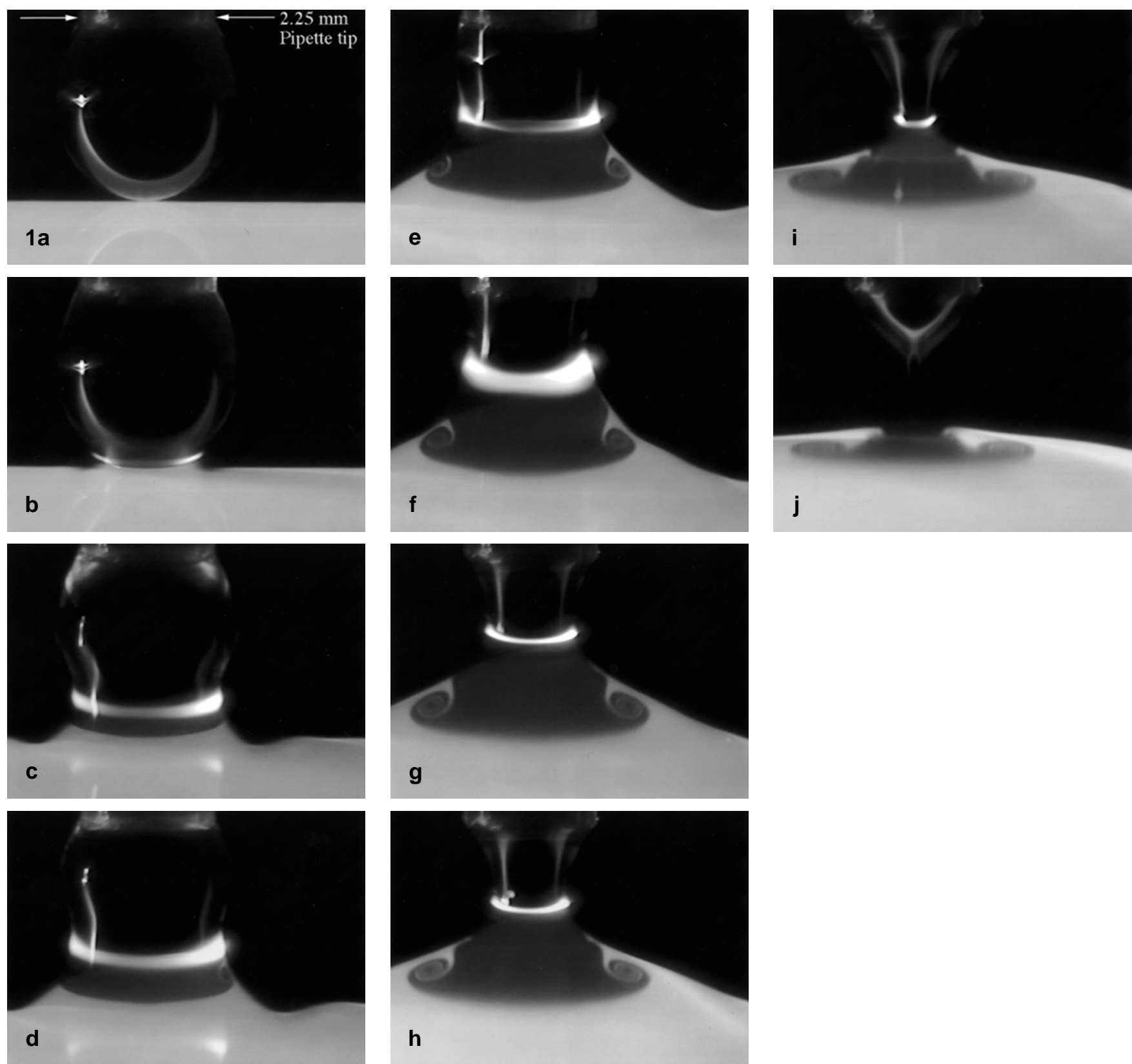

Fig. 1a-j. Sequence of photos depicting vortex ring formation. All exposures are $1 \mathrm{~ms}$. Start times of exposure are as follows (drop contact with water at $t=0$ ). $a$ Static photo just before contact; pipette tip location and size indicated; $b-0.8 \mathrm{~ms}$; contact occurred during exposure; $c 1.1 \mathrm{~ms}$; d $2.4 \mathrm{~ms}$; e $4.6 \mathrm{~ms}$; $f 6.7 \mathrm{~ms} ; g 8.4 \mathrm{~ms}$; $h 9.1 \mathrm{~ms} ; i 12.1 \mathrm{~ms}$; $j 13.7 \mathrm{~ms}$

Rood's vorticity flux relation, transformed into the coordinate system shown in Fig. 2, and neglecting a gravitational term, reads:

$v\left(\frac{\partial \omega_{\mathrm{z}}}{\partial r}\right)_{r=0}=-\frac{\partial u_{\mathrm{s}}}{\partial t}-u_{\mathrm{s}} \frac{\partial u_{\mathrm{s}}}{\partial s}$

For clarity, we restrict our analysis to the right-hand side of the images only; analogous results hold for the other side, with different signs. On the right side, observed vorticity is of the positive sign in the coordinate system used. We identify two distinct possible means of vorticity flux into the fluid based on Eq. (1).
As was mentioned above, a large surface-parallel acceleration $\left(\sim 100 \mathrm{~m} / \mathrm{s}^{2}\right)$ is observed in the fluid very near the free surface in the first one to two ms of the process. This acceleration is in the negative $s$ direction, rendering the quantity $\left(-\partial u_{\mathrm{s}} / \partial t\right)$ in Eq. (1) positive. Such is of the proper sign, then, to cause flux of positive vorticity into the liquid, as shown in Fig. 2.

Figure 3 depicts the coalescence process at a later state. We deduce from the images that the fluid in region $\mathrm{A}$ is moving in the positive $s$ direction, downward. It can also be observed in the images that the dyed pool fluid very near the free surface in region $\mathrm{B}$ (the "tendril") is moving in the negative $s$ direction, or upwards. This combination necessitates the existence of 


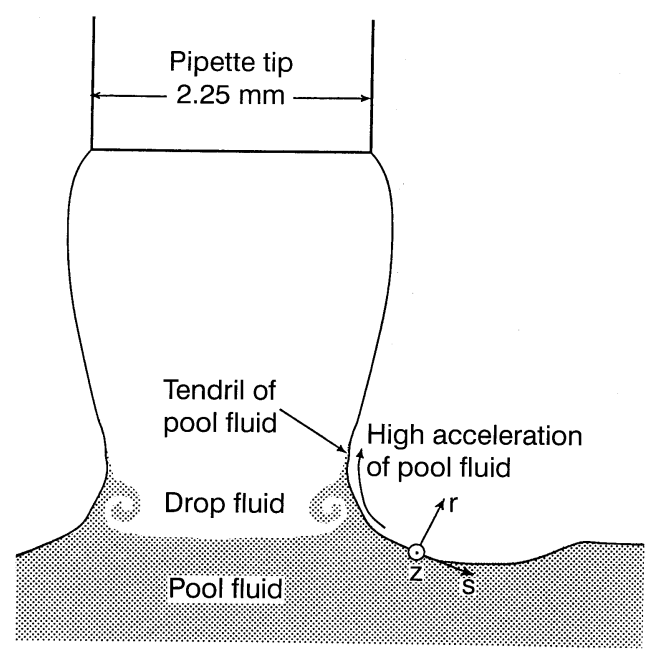

Fig. 2. Schematic of beginning of the ring formation showing tendril position and fluid acceleration

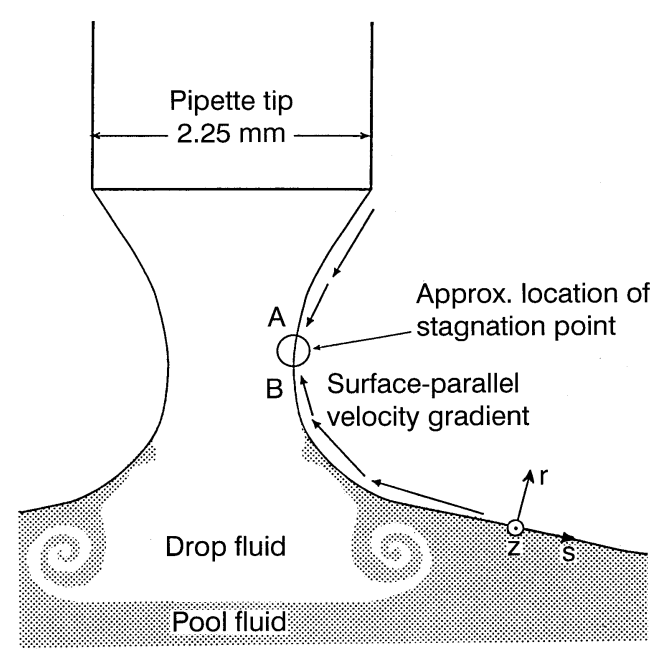

Fig. 3. Schematic of later stage of ring formation showing velocity gradient along surface

a stagnation point on the free surface at some location in between. Moreover, it implies that the quantity $\left(-u_{\mathrm{s}} \partial u_{\mathrm{s}} / \partial s\right)$ is positive in at least some portion of region $B$. This, too, is of the proper sign to induce positive vorticity flux into the fluid.

The speculative nature of these results must not be understated; in the absence of reliable quantitative data, they may only be regarded as tentative. They do, however, represent a plausible mechanism for the vorticity flux which so clearly must occur during the coalescence process.

\section{3}

\section{Scaling}

The experimental runs in which surface tension or drop diameter was varied served to corroborate the scaling law predictions described earlier. The characteristic time $\tau_{\star}$ of each run was taken to be the interval during which a fluid connection existed between the pool and the pipette. This time was easily identifiable with the use of the electrical circuit

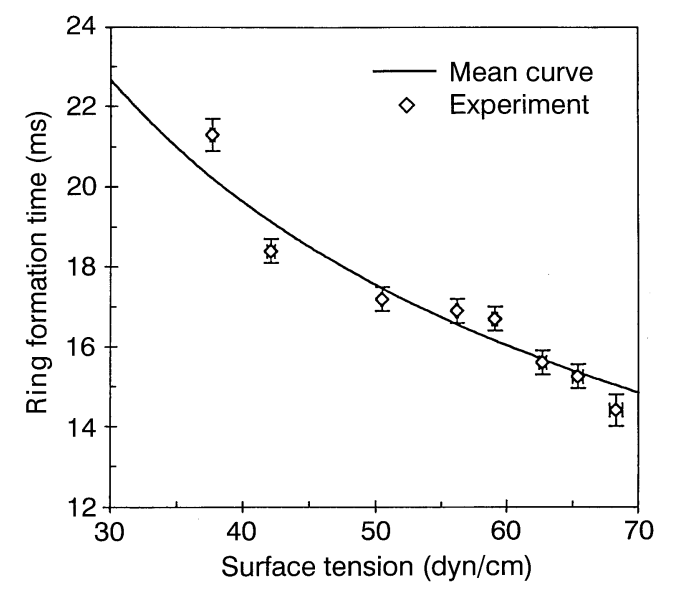

Fig. 4. Variation of ring formation time with fluid surface tension

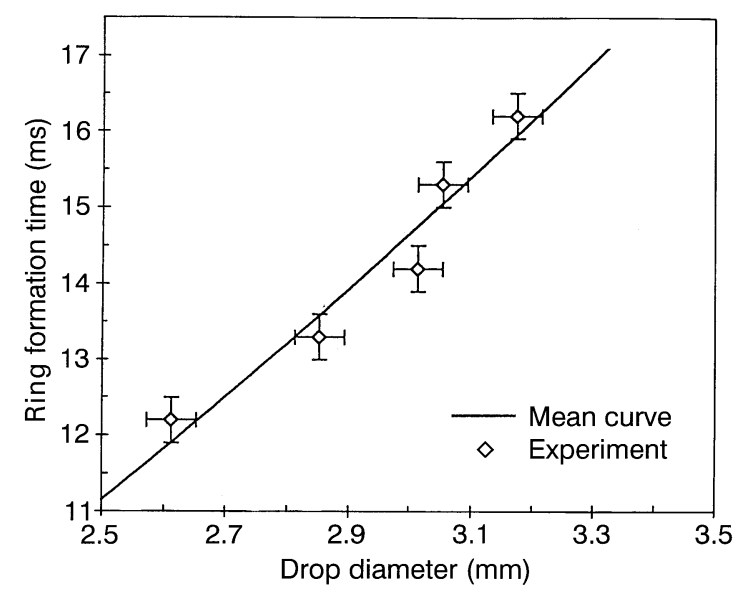

Fig. 5. Variation of ring formation time with drop diameter

described above. During this set of experiments, images were not recorded. For this reason, we did not use Fluorescein dye and hence avoided the minor (3\%) surface-tension differential between dyed and undyed fluid.

The scaling relations predicted above are borne out by experiment. The runs conducted with varying surface tension and diameter are summarized graphically in Figs. 4 and 5, respectively. Only one of the two quantities was varied at a time; the other was held constant. The "mean curves" plotted on the charts represent, for the surface tension case,

$\tau_{*}=K_{\sigma} \sigma^{-1 / 2}$

The multiplicative constant $K_{\sigma}$ was determined from the experimental data as the mean of

$K_{\sigma, i}=\tau_{\star i} \sigma_{i}^{1 / 2}$

taken over all of the points obtained. The diameter variation curve was determined in an analogous manner; the offset of the curve is zero. $K_{\sigma}$ was found to be $(124 \pm 4)$ dyn $\mathrm{ms} / \mathrm{cm}$, and $K_{L}$, the constant for the time-diameter relation, is $(0.72 \pm 0.02) \mathrm{ms} / \mathrm{mm}^{3 / 2}$. 
Because the droplets were slightly oblate, it is possible that the proper lengthscale of the drops is not their horizontal width, as was assumed above, but rather their vertical height. The data obtained was also analyzed with $L^{\prime}$ defined as the distance from the tip of the pipette to the surface of the pool, and a scaling proportionality constant $K_{L}^{\prime}$ found to be $(0.77 \pm 0.12) \mathrm{ms} / \mathrm{mm}^{3 / 2}$. The percentage standard deviation of $K_{L}(3 \%)$ is a factor of five lower than that for $K_{L}^{\prime}(16 \%)$. For this reason, it is felt that the proper lengthscale for scaling is the horizontal width of the drop.

A number of possible explanations exist for the less-thanperfect fit of the data to the power-law curve in Fig. 4. It is possible that the assumption of negligible gravitational potential energy, compared to surface energy, may be near its limit. This would be a consequence of the use of air and water as the two fluids involved. A simple calculation shows that the ratio of gravitational potential energy to surface energy in the drop just prior to contact with the pool is in the range of $15-25 \%$, depending on exact run conditions. Anilkumar et al. (1991), using two immiscible liquids rather than a liquid and a gas, operated at ratios of not more than $6 \%$. Nevertheless, the overall agreement of the trend of the data in Fig. 4 with that of the power law curve supports the notion that surface tension effects dominate the process in the regime examined.

In addition, the real effects of the slight oblateness of the droplets, and the presence of the pipette tip during coalescence are difficult to predict. The authors suggest that complicating effects such as these become more pronounced in their influences as fluid surface tension is lowered, and the absolute magnitude of the dominant force is similarly reduced. Such would allow other factors, constant from run to run, to exert a progressively greater influence. It is proposed that any effect these factors had on the data obtained was minor, due to the overall agreement of the data with scaling law predictions.

\section{5}

\section{Conclusions}

We have experimentally examined the formation of a vortex ring caused by the contract of a fluid drop with a pool composed of the same liquid. Photographs of the process reveal several physical characteristics of the vortex ring formation. We are able to qualitatively speculate on the source terms of vorticity flux through the free surface based on the images. A scaling law predicting the relationship between drop diameter, fluid surface tension, and a characteristic time process is shown to be valid. The assumptions inherent in the scaling law of negligible gravitational and viscous effects are thus justified for this case.

\section{References}

Anilkumar AV; Lee CP; Wang TG (1991) Surface-tension-induced mixing following coalescence of initially stationary drops. Phys Fluids A 3: 2587-2591

Chapman DS; Critchlow PR (1967) Formation of vortex rings from falling drops. J Fluid Mech 29: 177-185

Cresswell RW; Morton BR (1995) Drop-formed vortex rings - The generation of vorticity. Phys Fluids 7: 1363-1370

Hsiao M; Lichter S; Quintero LG (1988) The critical Weber number for vortex and jet formation for drops impinging on a liquid pool. Phys Fluids 31: 3560-3562

Peck B; Sigurdson L (1994) The three dimensional vortex structure of an impacting water drop. Phys Fluids 6: 564-576

Rogers WB (1858) On the formation of rotating rings by air and liquids under certain conditions of discharge. Amer J Sci, Second Ser 26: 246-258

Rood EP (1994) Interpreting vortex interactions with a free surface. J Fluids Eng 116: 91-94

Shankar PN; Kumar M (1995) Vortex rings generated by drops just coalescing with a pool. Phys Fluids 7: 737-736

Thomson JJ; Newall HF (1885) On the formation of vortex rings by drops falling into liquids, and some allied phenomena. Proc R Soc London 39: 417-436 